\title{
Urticaria aguda y tratamiento ambulatorio indicado en emergencia de dos hospitales de atención pediátrica.
}

Acute urticarial and outpatient treatment given in two pediatric care hospitals.

\author{
Mario Rolando Torres Madrid*
}

\section{RESUMEN}

Antecedentes: La urticaria aguda es una dermatosis frecuente, se calcula que ocurre en $20 \%$ de la población mundial. La tasa de prevalencia varía de acuerdo con la población de cada país entre 3.4 y 6.4\%. En Honduras en el Instituto Hondureño de Seguridad Social (IHSS), la urticaria aguda es la cuarta causa más frecuente de dermatosis en consulta externa dermatológica pediátrica. Objetivo: Determinar las características clínicas-sociodemográficas y el manejo en salas de Emergencia del Hospital Nacional Mario Catarino Rivas (HNMCR) y IHSS ambos hospitales en SPS, en el período enero 2016-julio 2017. Pacientes y Métodos: El diseño de la investigación fue transversal observacional, con alcance descriptivo. Se incluyeron 83 pacientes en edad pediátrica con diagnóstico de urticaria aguda, evaluados en las emergencias del IHSS y HNMCR. Resultados: El grupo etario y raza afectados fueron los lactantes menores $68.67 \%$ $(n=57)$ y raza mestiza $86.75 \%(n=72)$. No tenían antecedentes inmunoalérgicos $63.86 \%(n=53)$. El alimento relacionado con urticaria fue el huevo $9.64 \%(n=8)$ y entre los medicamentos; los antibióticos $23.08 \%(n=19)$. El tratamiento administrado con mayor frecuencia fue la doble terapia $65.21 \%(n=30)$ y se ingresaron 17 pacientes (20.48\%). Conclusión: El manejo terapéutico y los criterios de ingreso utilizado en el servicio de emergencia no siguen los lineamientos de guías internacionales.

* Residente de tercer año, Posgrado de Pediatría, Universidad Nacional Autónoma de Honduras en el Valle de Sula (UNAH-VS).

Dirigir correspondencia a: mrtorres9@hotmail.com

Recibido: 20 de diciembre 2017 Aprobado: 08 de enero 2018

\section{PALABRA CLAVE}

Urticaria, Angioedema, Niño.

\section{ABSTRACT}

Acute urticaria is a frequent dermatosis, it is estimated that it occurs in $20 \%$ of the world population. Prevalence rates vary according to the population of each country between 3.4 and $6.4 \%$. In Honduras at the IHSS Israel Salinas, acute urticaria is the fourth most frequent cause of pediatric dermatological outpatient dermatosis. The objective of the study was to determine the clinical-sociodemographic cha- racteristics and emergency room management in two Regional Hospitals of San Pedro Sula, in the period January 2016 July 2017. Patients and Methods: The design of the research was transversal observational, with descriptive sco- pe. We included 83 pediatric patients diagnosed with acute urticaria, evaluated in the emergencies of the Israel Salinas Honduran Social Security Hospital and Mario Catarino Rivas National Hospital. Results: the age group and affected race were the younger infants $68.67 \%(n=57)$ and the mestizo race $86.75 \%(n=72)$. They had no immunoallergic history $63.86 \% \quad(n=53)$. The food related to urticaria was the egg $9.64 \%$ $(n=8)$ and among the medications the antibiotics $23.08 \%(n=19)$. The treatment administered most frequently was double therapy $65.21 \%(n=30)$ and 17 patients were admitted (20.48\%). Conclusion: The therapeutic management and the criteria of admission used in the emergency service do not follow the guidelines of international guidelines. 


\section{KEYWORDS}

Urticaria, Angioedema, Children.

\section{INTRODUCCIÓN}

La urticaria aguda es una reacción limitada a la piel y mucosas que se caracteriza por lesiones tipo roncha, habón, pápula y mácula, la cual se presenta en un periodo de tiempo menor de seis semanas. Su etiología es variada teniendo como principal desencadenante alimentos (huevo, mariscos, cítricos), medicamentos (antibióticos y analgésicos), e infecciones (respiratorias altas, gastrointestinales y genitourinarias). Sus mecanismos etiopatogénicos también suelen ser múltiples. ${ }^{(1)}$ El diagnóstico es plenamente clínico, no existen marcadores específicos y el pilar del tratamiento se basa en antihistamínicos y esteroides sistémicos. ${ }^{(2)}$

Se estima que de $15 \%$ a $25 \%$ de la población general puede presentar este cuadro en algún momento de su vida. Un estudio danés, observacional, de cohorte, efectuado en niños con seguimiento desde el nacimiento hasta los seis años, reporta una prevalencia de urticaria de

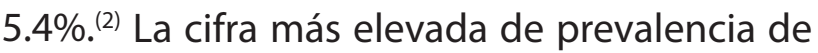
urticaria se ha documentado en niños preescolares atópicos: en el grupo placebo del estudio Early Prevention Asthma in Atopic Children reportando prevalencia de $42 \%$. $^{(3)}$

En una revisión retrospectiva del Hospital General de México se encontró que 187 de $1,913(9.7 \%)$ pacientes de la consulta externa de alergia en 2012 acudieron por urticaria. ${ }^{(4)}$

En Honduras en el IHSS Israel salinas, la urticaria aguda es la cuarta causa más frecuente de dermatosis de consulta externa dermatológica pediátrica con $13 \%$ de las consultas. ${ }^{(5)}$

En San Pedro Sula, no se han realizados estudios sobre urticaria en niños y no contamos con lineamientos de manejo, lo cual nos obliga a trabajar con información de otros países, que podría no concordar con nuestra realidad local. Se realizó el siguiente estudio con el objetivo de caracterizar clínica y sociodemográficamente la urticaria aguda y determinar si el manejo ambulatorio que actualmente brindamos en las salas de emergencia pediátrica es el adecuado.

\section{PACIENTES Y MÉTODOS}

El enfoque de la investigación fue cuantitativo con diseño transversal observacional de alcance descriptivo. El estudio se llevó a cabo en el área del filtro de las salas de emergencia del IHSS y HNMCR en el periodo de enero 2016-julio 2017, en pacientes con diagnóstico de urticaria aguda y que cuyos padres o encargados deseaban participar del estudio. Los criterios de exclusión fueron: padres o encargados que no desearon participar del estudio, pacientes con antecedentes de enfermedades sistémicas y pacientes bajo tratamiento por otra patología.

Se identificaron un total de 86 pacientes de los cuales se excluyeron 3 ya que los padres o encargados no desearon que sus hijos participaran del estudio.

El método de muestreo empleado fue no probabilístico mediante técnica de muestreo por conveniencia. La investigación se clasificó como categoría I (investigación sin riegos).

Para la recolección de los datos se contó con el consentimiento informado el cual fue autorizado por madre, padre o encargado del paciente, a quien se le garantizó la confidencialidad de la información que brindo.

El método y técnica de recolección de datos mediante entrevista directa en el momento de salir de la consulta en emergencia de ambos 
hospitales o el momento del alta si estuvieran hospitalizados. El formulario de recopilación de información constó de preguntas cerradas y abiertas sobre datos generales, antecedentes previos de urticaria, tiempo de inicio de la urticaria, determinación de la forma clínica de las lesiones, datos clínicos evolutivos de importancia, así como variables asociadas a exámen físico.

La terapia se clasificó como: monoterapia (antihistamínicos sistémicos), doble terapia (antihistamínicos sistémicos + esteroides sistémicos) y triple terapia (antihistamínicos sistémicos + esteroides sistémicos + esteroides tópicos).

Los resultados se ingresaron en una base de datos (Programa IBM.SPSS.Statistics. 23.0).

Para el análisis de los datos se utilizaron medidas de frecuencia y porcentaje. Se dividieron los pacientes en dos grupos, los que recibieron manejo ambulatorio y los que recibieron manejo hospitalario.

\section{RESULTADOS}

En la sección de datos generales, los resultados obtenidos reflejan que el sexo masculino se presentó con $51.60 \%(n=42)$ y sexo femenino $49.40 \%(n=41)$, con una relación masculino: femenino 1:1. El grupo etario se distribuyó en lactantes menores $68.67 \%(n=57)$, seguido de los pre escolares con $14.46 \%(n=12)$, lactantes mayores $8.43 \%(n=7)$ y escolares $8.43 \%(n=7)$.

En la tabla No.1 se presentan los antecedentes alérgicos de los pacientes estudiados. No presentaron antecedente alérgico en $63.86 \%$ $(n=53)$; entre los pacientes que si presentaron antecedentes destacaron rinitis alérgica $7.23 \%$ $(n=6)$, problemas respiratorios a repetición $6.02 \%(n=5)$ junto a alergia alimentaria $6.02 \%$ $(n=5)$.
Tabla No. 1: Historia médica de pacientes con Urticaria.

\begin{tabular}{lcr}
\hline Antecedentes alérgicos & $\mathbf{n}$ & Porcentaje \\
\hline Ninguno & 53 & $63.86 \%$ \\
Rinitis alérgica & 6 & $7.23 \%$ \\
$\begin{array}{l}\text { Infecciones respiratorias } \\
\text { a repetición }\end{array}$ & 5 & $6.02 \%$ \\
Alimentos & 5 & $6.02 \%$ \\
Asma bronquial/Sibilante & 4 & $4.80 \%$ \\
recurrente & 4 & $4.80 \%$ \\
Medicamentos & 3 & $3.51 \%$ \\
Dermatitis atópica & 3 & $3.71 \%$ \\
Prurigo insectos & 83 & $100 \%$ \\
TOTAL &
\end{tabular}

Fuente: Encuesta Urticaria, IHSS-HNMCR, 2016

Entre los factores desencadenantes de la urticaria aguda, $73.49 \%(n=61)$ de los pacientes no consumieron alimentos desencadenantes 24 horas previas al cuadro de urticaria; en la tabla No. 2 se enlista además los alimentos alergénicos consumidos las 24 horas previas a la urticaria, especialmente huevo 9.64\% $(n=8)$, cítricos $7.23 \%(n=6)$ y mariscos $6.02 \%(n=5)$.

Tabla No. 2: Historia de exposición a agentes desencadenantes de urticaria aguda 24 previas en las $\mathbf{2 4}$ horas previas.

\begin{tabular}{lrr}
\hline & $\mathbf{n}$ & $\mathbf{\%}$ \\
\hline Alimentos consumidos & & \\
Ninguno & 61 & 73.49 \\
Huevo & 8 & 9.64 \\
Cítrico & 6 & 7.23 \\
Mariscos & 5 & 6.02 \\
Lácteos & 2 & 2.41 \\
Frutos secos & 1 & 1.20 \\
Medicamentos administrados & & \\
Desconoce & 30 & 36.54 \\
Antibiótico & 19 & 23.08 \\
Ninguno & 18 & 21.87 \\
Analgésico & 14 & 17.31 \\
Anticonvulsivante & 2 & 1.20 \\
\hline
\end{tabular}

Fuente: Encuesta Urticaria, IHSS-HNMCR, 2016 
Los medicamentos relacionados con urticaria administrados 24 horas previas al cuadro de urticaria aguda se presentan también en la tabla No. 2. Se desconoce si se administró medicamentos $36.5 \%(n=30)$. La mayoría de los medicamentos administrados fueron automedicados y el paciente que consumió anticonvulsivante fue administrado por equivocación. Los medicamentos utilizados fueron los antibióti$\cos 23.08 \%(n=19)$ y analgésicos $17.31 \%(n=14)$.

Las formas de presentación de la urticaria aguda en los pacientes de ambos hospitales fueron la roncha $42.17 \% \quad(n=35)$, el habón $16.87 \%(n=14)$, la mácula $15.66 \%(n=13)$; en menor frecuencia pápula $12.05 \%(n=10)$ y otros tipos de lesiones $13.25 \%(n=11)$. Entre los otros tipos de lesiones hubo dermografismo, lesión vasculítica. Se agruparon los pacientes atendidos en las emergencias de ambos hospitales de acuerdo a su manejo como ambulatorios $79.5 \%(n=66)$ y manejo intrahospitalario $20.5 \%(n=17)$. A continuación se presentan los datos obtenidos de acuerdo al tipo de manejo.

La región topográfica en piel de los pacientes manejados ambulatoriamente afectada fue generalizada en $44.6 \%(n=29)$ seguido de cara $19.3 \%(n=13)$. (Ver tabla No. 3) Otros corresponden a afectación de genitales externos y mucosa oral.

Tabla No. 3: Región topográfica en piel afectada en pacientes ambulatorios.

\begin{tabular}{lrr}
\hline Localización topográfica & $\mathbf{n}$ & \% \\
\hline Cara & 13 & 19.3 \\
Tórax anterior & 6 & 8.4 \\
Tórax posterior & 2 & 2.4 \\
Miembros superiores & 5 & 7.2 \\
Miembros inferiores & 2 & 3.6 \\
Generalizado & 29 & 44.6 \\
Otro & 9 & 14.5 \\
TOTAL & 66 & 100 \\
\hline
\end{tabular}

Fuente: Encuesta Urticaria, IHSS-HNMCR, 2016
Sobre el tratamiento ambulatorio brindado en la emergencia de ambos hospitales, la doble terapia fue el tratamiento utilizado para la urticaria aguda $65.21 \%(n=43)$, seguido de monoterapia $26.06 \%(n=17)$ y $8.69 \%(n=6)$ utilizó triple terapia. El tiempo de la terapia indica que se utilizó fue de 3 días $37.93 \% \quad(n=25)$ seguido de, 5 a 7 días en un 34.48\% $(n=23)$, de 3 a 5 días $17.24 \%(n=11)$ y solo un $10.34 \%$ $(\mathrm{n}=7)$ lo utilizo más de 7 días.

En la Tabla No. 4 se presenta las zonas afectadas por la Urticaria Aguda en los pacientes ingresados $(n=17)$ en ambas instituciones $47.06 \%$ $(\mathrm{n}=8)$ presentaron lesiones topográficas generalizada, en cara $23.53 \%(n=4)$, tórax anterior $11.76 \% \quad(n=2)$, miembros superiores $5.88 \%$ $(n=1)$ y otro $11.76 \%(n=2)$. La distribución por grupo etario se presentó en lactantes menores $76 \%(n=13)$, lactante mayor $5.88 \%(n=1)$ y preescolar $17.66 \%(n=3)$.

Tabla No. 4: Región topográfica en piel afectada en pacientes ingresados.

\begin{tabular}{lcr}
\hline Área topográfica afectada & $\mathbf{n}$ & \multicolumn{1}{c}{$\%$} \\
\hline Cara & 4 & 23.53 \\
Tórax anterior & 2 & 11.76 \\
Miembros superiores & 1 & 5.88 \\
Generalizado & 8 & 47.06 \\
Otro & 2 & 11.76 \\
TOTAL & 17 & 100 \\
\hline
\end{tabular}

Fuente: Encuesta Urticaria, IHSS-HNMCR, 2016

\section{DISCUSION}

Los antecedentes inmunoalérgicos se encuentran sumamente relacionados con urticaria aguda (UA), cerca del 40 a $50 \%$ de los pacientes presentan dichos antecedentes. ${ }^{(6,7)}$ Además, los medicamentos y los alimentos son los principales desencadenantes en la edad pediátrica, especialmente los antibióticos, analgésicos, antiparasitarios y anticonvulsivantes. Los alimentos relacionados con la UA son huevo, cítricos, mariscos y frutos secos. ${ }^{(8-10)}$ 
Giubi R, en su estudio realizado sobre Urticaria aguda y angioedema: etiología y características clínicas, encontró entre los pacientes estudiados presentaron antecedentes personales de atopia en un $29 \%$ y familiares en un $17 \%$, los pacientes que presentaron urticaria medicamentosa fueron $23 / 72$, de estos el $61 \%$ fue por analgésicos (AINES), en relación con los alimentos desencadenantes de urticaria el huevo se presentó en $40 \%$ en menores de 2 años y leche de vaca en $20 \%$. ${ }^{(11)}$

En el HNMCR y IHSS se observó que el 36.14\% de los pacientes presentaron antecedentes inmunoalergicos entre ellos rinitis alérgica, infecciones respiratorias altas, alergia a alimentos, asma bronquial/sibilante recurrente, dermatitis atópica y prúrigo por insectos. Esto es consecuencia de la transgresión alimentaria a la que son expuestos los niños a temprana edad. En otro estudio realizado en el HNMCR sobre prácticas de alimentación se documentó las prácticas inadecuadas de alimentación que tienen las madres de los lactantes en San Pedro Sula, como por ejemplo, con introducción del jugo de naranja desde los 15 días de vida, inicio de la ablactación con cereal, sopa Maggie antes de los 3 meses, inicio de leche entera a partir de los 7 días de vida, entre otros. ${ }^{(12)}$ La exposición a estos alimentos marca el inicio de la marcha atópica con la alergia a alimentos, dermatitis atópica, rinitis alérgica y posteriormente asma en la niñez y adultez temprana.

Los medicamentos como causa de urticaria en edad estuvo presente en $42.31 \%$ de la población, entre ellos antibióticos y analgésicos. Por otro lado, el $26.52 \%$ de la población presentó urticaria por alimentos como desencadenante. Los alimentos desencadenantes fueron huevo, cítricos, mariscos, lácteos y frutos secos.

Aunque la urticaria puede desencadenarse por una gran diversidad de factores, ya previamente descritos, y puede tener varias manifestaciones clínicas, el tratamiento de todas sus formas sigue los mismos principios. Según la Guía Mexicana para el Diagnóstico y el Tratamiento de la Urticaria se pueden estipular tres enfoques fundamentales que deben considerarse en cada paciente. ${ }^{(13)}$

\section{A. Evitar el estímulo desencadenante. Elimi- nación o tratamiento del estímulo desencade-} nante o de la causa. La prioridad en el tratamiento es la eliminación del agente causal, del estímulo o del antígeno, porque si se logra esto el resultado será la curación. Sin embargo, a menudo se desconoce el estímulo desencadenante exacto. En urticaria aguda los desencadenantes más frecuentes son alimentos, medicamentos e infecciones virales. ${ }^{(13)}$

\section{B. Inhibir la liberación de los mediadores de las células cebadas: Los fármacos más prescri-} tos para la inhibición de las células cebadas son los corticoesteroides. En el caso de la UA, un tratamiento corto con corticoesteroides sistémicos puede ser útil para reducir la duración de la enfermedad. Los esteroides sistémicos actúan al unirse a su receptor en el citosol de las células, reduciendo la actividad del NFKB (Nuclear Factor Kappa B), ${ }^{(14,15)}$ una proteína intraceluar proinflamatoria, al igual que lo hace con citocinas proinflamatorias, como la interleucina (IL) 1, IL-2, IL-3, IL-5, IL-6, IL-8, IL-12, Factor de Necrosis Tumoral alfa (TNF-alpha), Interferón gamma (IFN-gamma) y factor Estimulante de Colonias de Granulocitos y Macrófagos (GM-CSF5). ${ }^{(16)}$

Los esteroides sistémicos ocupan un lugar en el tratamiento de la UA o de las agudizaciones, donde se recomienda como tratamiento oral de corta duración (prednisona oral de 0.3 a 0.5 $\mathrm{mg} / \mathrm{kg}$ durante cinco a siete días o metilpredni- 
solona $1 \mathrm{mg} / \mathrm{kd}$ /día). Debido a que la urticaria es una enfermedad sistémica, no hay lugar para el tratamiento tópico con corticoesteroides. ${ }^{(17,18)}$

\section{Impedir la acción de los mediadores. Impo-} sibilitar la acción de los mediadores de las células cebadas sobre el tejido blanco: ${ }^{(3)}$ Casi todos los síntomas de la urticaria son mediados por histamina al fijarse a los receptores $\mathrm{H} 1$, que se localizan en las terminaciones nerviosas y en las células endoteliales. ${ }^{(19)}$ Por ello, los antihistamínicos sistémicos son el pilar terapéutico en este padecimiento. ${ }^{(20)}$

En cuanto a los antihistamínicos $\mathrm{H} 1$ orales para el tratamiento de la urticaria aguda, se recomienda la prescripción de antihistamínicos $\mathrm{H} 1$ orales de segunda generación, ya que estos no son sedantes y tienen efectos anticolinérgicos, como sequedad de boca y retención de orina y el $30 \%$ en su mayoría no atraviesan la barrera hematoencefálica como lo hacen los de primera generación. Los antihistamínicos de segunda generación también ejercen leves efectos antiinflamatorios, como la liberación de citocinas a partir de basófilos y células cebadas.

Para el tratamiento de la UA en pacientes que no mejoran con las dosis recomendadas o en pacientes que tienen síntomas moderados-graves se podría duplicar o cuadruplicar la dosis habitual de algunos antihistamínicos $\mathrm{H} 1$ orales de segunda generación, pero la recomendación es débil y la calidad de evidencia baja.

En el IHSS y HNMCR mostró que los pacientes atendidos en la sala de ambas emergencias pediátricas, 83 pacientes en su totalidad, 66 de ellos se manejaron ambulatoriamente, brindándoseles: doble terapia (esteroides sistémicos y antihistamínicos sistémicos), monotera pia (antihistamínicos sistémicos) y triple terapia (esteroides sistémicos, antihistamínicos sistémicos y esteroides tópicos). El tiempo de prescripción de estos fue de 3 días en su mayoría. Estos resultados muestran la falta de un protocolo de manejo de esta patología ya que el manejo que actualmente se brinda en ambas instituciones no es el adecuado. No se logró estudiar a fondo el tipo de antihistamínicos y esteroides sistémicos administrados ya que en la mayor parte del tiempo en ambas instituciones no se logra contar con el cuadro básico de medicamentos.

La mayoría de las urticarias no presenta sintomatología sistémica, menos de 3\% de urticaria puede llegar a complicarse.(4) Entre ellas se puede presentar la Urticaria Vasculitis que se manifiesta con habones eritematosos recurrentes por más de cuatro a seis semanas, que duran más de 24 horas y desaparecen dejando hiperpigmentación residual. Según los niveles de complemento esta entidad puede subdividirse en urticaria vasculítica normocomplementémica e hipocomplementémica. Aquellos pacientes con hipocomplementemia tienen mayor riesgo de desarrollar compromiso multiorgánico y frecuentemente desarrollan Lupus Eritematoso Sistémico durante el seguimiento, especialmente los que cursan con anticuerpos anti-C1q. La Urticaria gigante o angioedema: (edema que no deja fóvea, no pruriginosa, y generalmente indolora, aunque puede producir sensación de quemazón). Las lesiones comprometen a la dermis profunda y el tejido celular subcutáneo y se considera la extensión profunda de la urticaria. También puede afectarse el tracto respiratorio y gastrointestinal. Dentro de sus complicaciones se encuentran choque anafiláctico y edema laríngeo.

Los criterios de ingreso de UA según los lineamientos internacionales son urticaria gigante, 
ingresados en el IHSS y HNMCR. Se ingresaron un total de 17 pacientes entre los cuales la región topográfica afectada fue generalizada (más de dos regiones topográficas afectadas) (47.06\%). En cuanto a la distribución por grupo etario de los pacientes ingresados, el grupo más afectado fue los lactantes menores en $76 \%$. Por tanto, probablemente el criterio que se utilizó en el área de emergencia para decidir el ingreso de los pacientes con UA fue la edad de presentación y no se utilizó el área topográfica ya que en los pacientes no hospitalizados $(n=66)$ el $44.6 \%(n=29)$ presentaron área topográfica generalizada y no se hospitalizaron.

En conclusión, el manejo actual que se brinda a los pacientes pediátricos con urticaria aguda en IHSS y HNMCR no es el adecuado ya que no sigue los lineamientos de las guías internacionales.
Se recomienda la elaboración de Guía de Manejo Nacional Ambulatoria y Hospitalaria de la Urticaria Aguda, y garantizar la disponibilidad de los medicamentos necesarios para su manejo. Además, de promover la educación de la población sobre prácticas de alimentación adecuadas para la edad de los niños.

\section{AGRADECIMIENTO}

Les agradezco a mis asesores de tesis Dr. Héctor Caballero y Dr. Gerardo García por guiarme y brindar su conocimiento, apoyo y paciencia para la realización de la tesis y artículo original.

\section{REFERENCIAS BIBLIOGRÁFICAS}

1. Sánchez-Borges M, Asero R, Ansotegui IJ, Baiardini l, et al. Diagnosis and treatment of urticaria and angioedema: a worldwide perspective. World Allergy Organ J 2012; 5:125-147.

2. Sánchez-Saldaña $L$, Ponce-Rodríguez $M, 2$, Cabanillas-Becerra J, urticaria aguda, Dermatol PerU 2012; vol 22(1).

3. Zuberbier T, Balke M, Worm M, Edenharter G, Maurer M. Epidemiology of urticaria: a representative cross-sectional population survey. Clin Exp Dermatol 2010; 35: 869873.

4. Pite H, Wedi B, Borrego LM, Kapp A, Raap U. Management of childhood urticaria: current knowledge and practical recom- mendations. Acta dermato-venereológica. 2013; 93: 500-508.

5. Cariño-Cartagena D, Velasco-Medina A, Fernández de Córdova-Aguirre J, Arroyo-Cruz M, Velázquez-Samano G. Descriptive study of urticaria and angioedema in Allergy and Immunology Department of Hospital General de Mexico Dr. Eduardo Liceaga. Ann Allergy Asthma Immunol 2013; 111:349.

6. Ferrufino J,Euceda, Caballero H. Caracterización epidemiológica y clínica de las cinco dermatosis más frecuentes en consulta externa pediátrica dermatológica. Acta Pediátrica Hondureña, Vol. 6, No. 2 /octubre 2015 a marzo 2016: 473-478. 
7. Gaig P, Olona M, Munoz Lejarazu D, Caballero $M T$, et al. Epidemiology of urticaria in Spain. J Invest Allergol Clin Immunol 2004; 14: 214-220.

8. Comert S, Celebioglu E, Karakaya G, Kalyoncu AF. The general characteristics of acute urticaria attacks and the factors predictive of progression to chronic urticaria. Allergol Immunopathol 2013;41:239-245.

9. Cariño-Cartagena D, Velasco-Medina A, Fernández de Córdova-Aguirre J, Arroyo-Cruz M, Velázquez-Samano G. Descriptive study of urticaria and angioedema in Allergy and Immunology Department of Hospital General de Mexico Dr. Eduardo Liceaga. Ann Allergy Asthma Immunol 2013; 111:349.

10. Asero R, Bavbek S, Blanca M, Blanca-Lopez $\mathrm{N}$, et al. Clinical management of patients with a history of urticaria/ angioedema induced by multiple NSAIDs: an expert panel review. Int Arch Allergy Immunol 2013; 160:126-133.

11. Sánchez-Saldaña $L$, Ponce-Rodríguez $M, 2$, Cabanillas-Becerra J, urticaria aguda, Dermatol PerU 2012; vol 22(1).

12. Prado LP, Aldana AM, Corea DM, Cruz RE. Prácticas de alimentación en lactantes del Hospital Nacional Dr. Mario Catarino Rivas. Act Ped Hon. 7(1): 538-547.

13. Larenas-Linnemann D, Medina-Ávalos M, Ortega-Martell J y col, Guía Mexicana para el Diagnóstico y el Tratamiento de la Urticaria. Revista Alergia México, Volumen 61, Suplemento 2, 2014.
14. Simons FE, Simons KJ. Histamine and H1 -antihistamines: celebrating a century of progress. J Allergy Clin Immunol 2011; 128:1139-1150.

15. Greaves MW. Recent advances in pathophysiology and current management of itch. Ann Acad Med 2007;36:788-792.

16. Yanai K, Zhang D, Tashiro M, Yoshikawa T, et al. Positron emission tomography evaluation of sedative properties of antihistamines. Expert Opinion on Drug Safety 2011; 10:613-622.

17. Asero AT. Usefulness of a short course of oral prednisone in antihistamine-resistant chronic urticaria; a retrospective analysis. J Investig Allergol Clin Immunol 2010; 5:386-390.

18. Asero AT, Cugno M. Treatment of refractory chronic urticaria: Current and future therapeutic options. Am J Clin Dermatol 2013:481-488.

19. Yanai K, Zhang D, Tashiro M, Yoshikawa T, et al. Positron emission tomography evaluation of sedative properties of antihistamines. Expert Opinion on Drug Safety 2011; 10:613-622.

20. Simons FE, Simons KJ. Histamine and H1 -antihistamines: celebrating a century of progress. J Allergy Clin Immunol 2011; 128:1139-1150. 